\title{
Social support networks and type of neurotic symptom among adults in British households
}

\author{
T. S. BRUGHA, ${ }^{1}$ Z. MORGAN, P. BEBBINGTON, R. JENKINS, G. LEWIS, \\ M. FARRELL AND H. MELTZER
}

From the Department of Psychiatry, University of Leicester; Department of Psychiatry and Behavioural Sciences, University College London Medical School, Institute of Psychiatry and Office for National Statistics, London; and Department of Psychological Medicine, University of Wales College of Medicine, Cardiff

\begin{abstract}
Background. Current knowledge about associations between psychosocial factors and nonpsychotic symptoms provide little information about their relationship to specific types of neurotic symptoms such as symptoms of fatigue, worry, phobic anxiety and obsessional symptoms.

Method. The British National Survey of Psychiatric Morbidity was based on a cross-sectional random sample of 10108 householders. Neurotic symptoms were established by lay interviewers using the revised fully structured Clinical Interview Schedule (CIS-R). Subjects were asked about perceived social support, the size of their close primary social network and sociodemographic attributes. To assess possible associations between specific types of neurotic symptoms and psychosocial risk factors multivariate Huber logistic models (a modified form of repeated measures design modelling) was used taking account of correlation between symptom types and sampling design including clustering.
\end{abstract}

Results. After controlling for sociodemographic factors the risk of having a high total CIS-R score $(\geqslant 12)$ was approximately doubled for both types of poor social functioning. Specific types of neurotic symptoms were associated both with a small primary group and with inadequate perceived social support. Depression, depressive ideas and panic symptoms had a higher prevalence in multivariate models. Poverty was associated with low support.

Conclusions. Associations with deficiencies in social support and self-reported neurotic symptoms are better explained by symptom type and in particular by depression than by the total number of symptoms. If confirmed by longitudinal study findings this knowledge could be used to inform the development of interventions to improve social support in order to reduce specific neurotic symptom types.

\section{INTRODUCTION}

A major purpose of community psychiatric surveys is to examine those associations between risk factors and psychiatric disorders in the general population that have been found in patients in receipt of clinical services. Deficiencies in social support and the protective effect of social support have been the subject of

${ }^{1}$ Address for correspondence: Professor T. S. Brugha, University of Leicester, Section of Social and Epidemiological Psychiatry, Department of Psychiatry, Brandon Mental Health Unit, Leicester General Hospital, Gwendolen Road, Leicester LE5 4PW. investigation in clinical and small community surveys since the theoretical case for conducting such investigations was set out (Cassel, 1976; Cobb, 1976; Tolsdorf, 1976; Henderson, 1977). Progress since has been documented in various reviews (House et al. 1988; Brugha, 1995).

Interest in social support rests not only upon its potential importance as a cause of ill health but in particular upon the possible public health benefit of developing interventions to modify risk and thus to prevent mental disorders in the general population (Mrazek \& Haggerty, 1994; 
Harris et al. 1999). Valuable information about the role of social support in the inception of psychiatric disorder comes from communitybased prospective studies (Brown et al. 1986).

Most research focuses on either symptoms of depression or on non-specific psychological distress in accordance with a dimensional view of neurosis (Goldberg \& Huxley, 1992). This is the most parsimonious model of neurosis disturbance, but it might yet be the case that the distribution of specific neurotic disorder offers a better description of clinical reality. However, there is insufficient comparative information about the nature of risk factors for the development of specific forms of symptomatology such as fatigue, worry and nervous tension, phobic anxiety and obsessive-compulsive symptoms. Psychological and other biological mechanisms underlying these may differ in relation to social risk factors. Such information would provide potentially valuable public health information as a forerunner to the development and evaluation of targeted prevention and health promotion interventions (Mrazek \& Haggerty, 1994). By way of illustration, suppose the risk factor 'small social network' primarily predicted social anxiety (and secondarily depression) the development of an intervention to prevent depression could begin by considering such a mechanism.

The development of a fully structured revised Clinical Interview Schedule (CIS-R) (Lewis et al. 1992) from an earlier semi-structured clinician administered interview (Goldberg et al. 1970) retained the capacity for 'bottom-up' collection of data on a wide range of types of neurotic symptoms (Table 1). A recent comparison in a British household population sample of the CIS-R with a clinician administered semistructured interview showed acceptable agreement for severity of depression (Brugha et al. $1999 a$ ). This suggests that although the CIS-R is a self-report measure it can be used in large-scale surveys to examine specific symptom groupings without the direct involvement of additional lengthy and costly clinical assessments.

Large scale national surveys offer certain advantages (as well as disadvantages): greater statistical power required to study rarer types of symptoms and greater generalizability by using probability samples with adjustment for clustering, non-response and other differences between
Table 1. The 14 sections of the revised Clinical Interview Schedule (CIS-R)

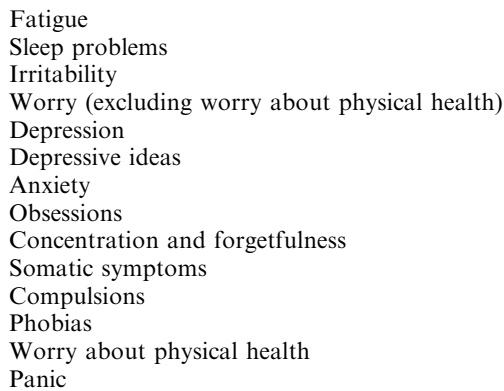

samples and the population they are drawn from. Examining the full range of the commoner forms of symptoms of psychiatric disorder in the community without imposing diagnostic rules developed in specialist clinical services may also be informative. A particular reason for this approach is that the use of non-clinician (i.e. self-report) measures to establish formal diagnostic rules has yielded evidence of poor agreement for diagnostic grouping in comparisons with clinician-administered assessments (Brugha et al. 1999a); but this comparison shows that agreement for overall severity of, for example, depression is more acceptable (Brugha et al. 1999 b).

The National Survey of Psychiatric Morbidity (Jenkins et al. $1997 a, b$ ) provided fully structured measures of psychiatric symptoms (and disorder), together with social and demographic information that could be used to examine associations with social support after controlling for possible confounding variables. Predictors of deficient social support were also estimated. We hypothesized that adults aged 16 to 64 with deficient social support and social networks would have higher rates of neurotic symptoms. In line with the more parsimonious dimensional model of neurosis we also hypothesized that this association would not vary significantly when the type of neurotic symptom is taken into account.

\section{METHOD}

\section{Sampling}

The methods used in the household survey of psychiatric morbidity have been described in 
greater detail elsewhere (Meltzer et al. $1995 a$, $b, c$; Jenkins et al. $1997 a, b)$. The field-work was carried out in 1993. The sample was drawn using the small area Postcode Address File as the sampling frame (Wilson \& Elliot, 1987). Two hundred postal sectors covering all of Great Britain, except the Highlands and Islands of Scotland, were selected at random with a probability proportional to the number of delivery points. Within each of these, 90 delivery points were randomly selected to generate a sample of 18000 delivery points. From among these addresses, private households with at least one person aged 16 to 64 were identified. In all, 15765 private households were identified: 12730 adults were eligible for interview, of whom 10108 agreed to take part in the survey. Only one eligible adult (i.e. aged 16-64) was interviewed in each household, randomly selected by the Kish grid method (Kish, 1965). Probability weights were used to take account of this sampling procedure and also to render the sample representative of the demographic characteristics of the population in Britain.

\section{Interviewers and interviewer training}

The interviews were carried out by two hundred interviewers from the Social Survey Division fieldwork team of the British Office for Population Censuses and Surveys (now the Social Survey Division of the Office for National Statistics). These interviewers had a minimum of 3 years' prior interviewing experience and attended for a 1 day training programme in the use of the survey instruments, including the revised Clinical Interview Schedule (CIS-R) (Lewis et al. 1992). Field-work was closely monitored by supervisors in the field and by headquarters staff.

\section{Assessment}

Neurotic psychiatric symptoms and disorders was assessed using the CIS-R. This has advantages that made it suitable for the National Survey. It is designed to be administered by non-clinically trained interviewers, and this was straightforward for the experienced ONS interviewers used in the survey. Moreover, the interview itself is relatively short (ranging from 15 to $30 \mathrm{~min}$ ) compared with other methods of assessment.
The CIS-R was used to establish an overall symptom score. It has 14 sections (panel) scored from 0 to 4 (except for the section on Depressive Ideas, which has a maximum score of 5). Within each section symptoms are regarded as severe if they have a score of $\geqslant 2$. A summed (total) score is also available that can in theory range between 0 and 57. The overall threshold score for significant psychiatric morbidity is 12 (Lewis et al. 1992).

Perceived social support was assessed from respondents' answers to seven questions taken from the 1987 Health and Lifestyle survey (Cox et al. 1987). These questions were also asked in the Health Survey for England (Breeze et al. 1994), providing additional comparative data. The seven questions take the form of statements which individuals could say were not true, partly true, or certainly true of their family and friends. Scores of 1-3 were obtained for each question and overall scores ranged from 7 to 21 . The maximum score of 21 indicated no lack of social support, scores of 18 to 20 indicated a moderate lack of social support and scores of $\leqslant 17$ showed that individuals perceived a severe lack of social support. The seven statements are:

There are people I know - amongst my family or friends -

(1) who do things to make me happy;

(2) who make me feel loved;

(3) who can be relied on no matter what happens;

(4) who would see that I am taken care of if I needed to be;

(5) who accept me just as I am;

(6) who make me feel an important part of their lives;

(7) who give me support and encouragement.

The size of an individual's primary support group was assessed with a series of questions focused on the numbers of friends and relatives (aged $\geqslant 16$ ) respondents felt close to (Brugha et al. 1987). Data were collected about three groups of people:

(1) adults who lived with respondents that respondents felt close to;

(2) relatives who did not live with respondents that they felt close to;

(3) friends or acquaintances who did not live with respondents but who would be described as close or good friends. 
Close friends and relatives form an individual's 'primary support group' (Henderson et al. 1978; Brugha et al. 1982); adults with a total primary group size of three people or fewer have previously been shown to be at greatest risk of psychiatric morbidity (Brugha et al. 1993).

Information was sought about variables that might confound in the association between neurotic disorder and social support deficits. Respondents completed questions covering employment status, type of family unit (household composition), long-standing physical ill health, location in a rural, semi-rural versus an urban area (Paykel et al. 2000) and access to a car. Employment status covered four groups: working full-time; working part-time; unemployed; and economically inactive. Because only that part of the sample who were classified as having a mental disorder were asked specific questions about their financial circumstances, the availability of a car was used as a proxy for income. We have used this variable effectively before (Lewis et al. 1998). The type of family unit was divided into five categories: one person family units; couples with children; couples without children; single-parent households; and, respondents living with parents (Meltzer \& Gill, 1993). Long-standing ill health was established by asking respondents if they suffered from any long-standing illness, disability or infirmity (Meltzer \& Gill, 1993). All adults interviewed in the survey were asked whether they had experienced one of 11 stressful life events during the past 6 months because such events may confound the association between social support deficits and psychiatric disorders (Brugha et al. $1990 a$ ). The events and problems were taken from the List of Threatening Experiences (Brugha et al. 1985). They were: personally suffering a serious illness; injury or assault; a close relative suffering a serious illness, injury or assault; death of a parent, spouse/partner, child, brother or sister; death of a close family friend or another relative; marital separation/break-up of steady relationship; serious problem with a close friend, neighbour or relative; redundancy/ sacking from job; unsuccessfully seeking work for more than 1 month; major financial crisis, such as losing the equivalent of 3 months income; problems with the police involving a court appearance; something valued being lost or stolen. In this paper, we examine stressful life events in terms of the presence of one or more events experienced in the 6-month period prior to interview.

\section{Analysis}

Analyses were based on all respondents who were directly interviewed in the National Survey: 271 subjects assessed by proxy were not included, as there were no CIS-R data on them. Analyses were based on weighted data performed using the survey commands in STATA 6.0 (STATA Corp, 1999), which gives more accurate precision of parameter estimates with the use of survey data incorporating probability weights to take account of sampling design including geographic clustering. We estimated the population proportion with two or more items endorsed within each neurotic symptom type according to the level of perceived social support and the grouped primary group size. We used logistic regression (for survey data) to examine sociodemographic predictors of deficient social support. A total CIS-R score of $\geqslant 12$ was employed as the dependent variable in a logistic regression model with social support, other sociodemographic factors and recent threatening life events as predictors. Standard statistical methods could not be used to investigate the association between social support networks and non-psychotic symptoms individually, as they are non-independent. For example you can not build a prediction model for fatigue in the absence of other neurotic symptoms; ignoring this will almost certainly lead to poor estimates of the standard errors. The problem is similar to that of a multiple (repeated) measures outcome design. Therefore, we used multivariate Huber logistic regression to analyse data for all 14 symptoms simultaneously, in an effort to establish whether or not social support networks are commonly associated with all symptoms or whether or not social support networks are selectively associated with particular symptoms. We employed XTGEE (STATA Corp, 1999) as suggested and used by (Zahner \& Daskalakis, 1997; Horton et al. 1999), specifying the within-group correlation structure, and obtained estimated odds ratios and $95 \%$ confidence intervals for multivariate Huber logistic models (robust variance estimater). The estimates presented are adjusted for clustering on each individual (i.e. correlated 
Table 2. Presence of two or more items endorsed within each neurotic symptom type according to size of primary network group $(\mathrm{N}=9837)$

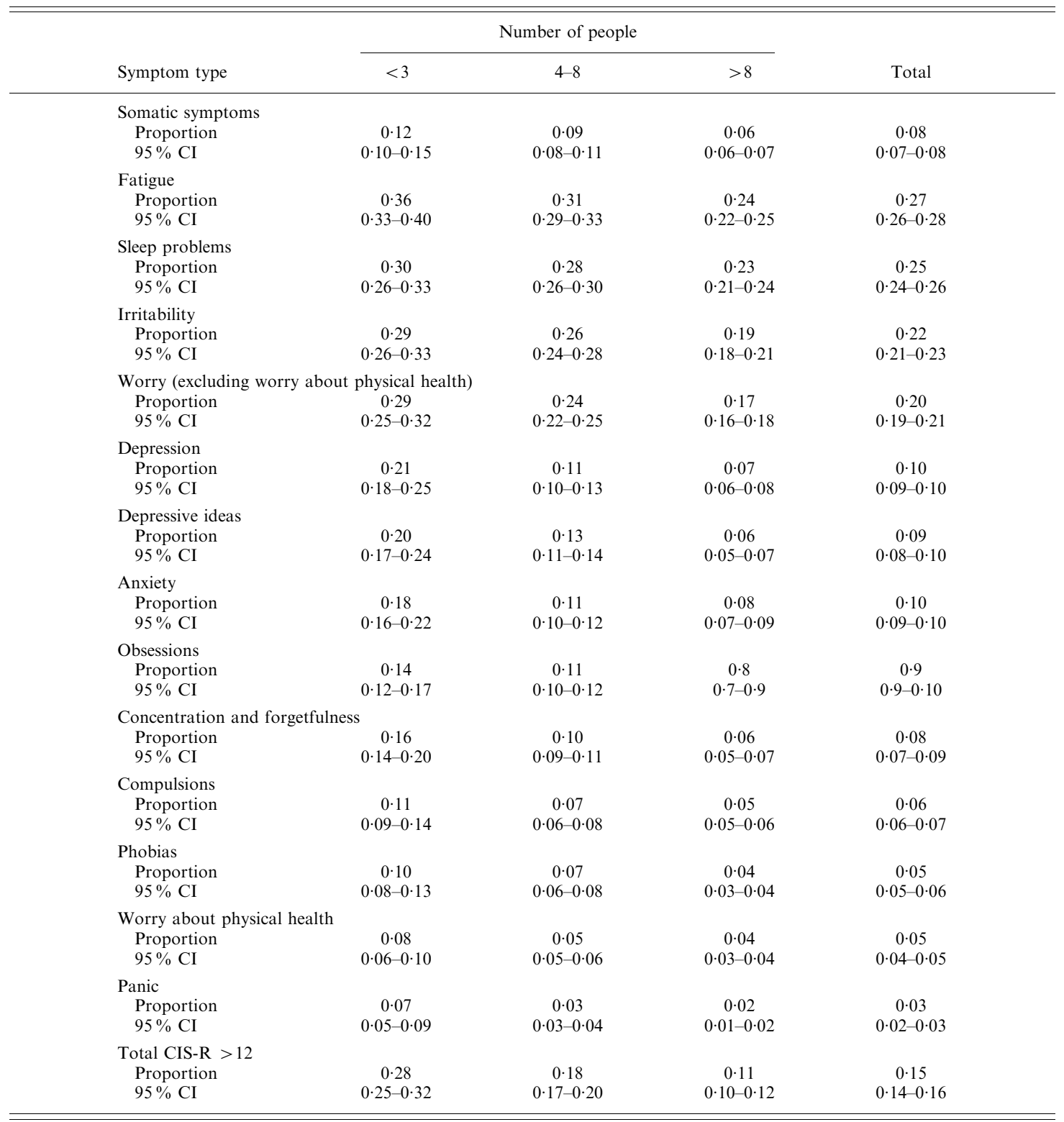

observations) and also incorporate sampling weights (full details available from the authors).

\section{RESULTS}

Based on a series of univariate analyses every type of neurotic symptom was highly statistically significantly associated with deficient social support (Tables 2 and 3). Typically persons with the most severe form of support deficit were twice as likely to have a particular symptom type when compared with the population as a whole. There was a suggestion that depressive symptoms and cognitions were more strongly 
Table 3. Presence of two or more items endorsed within each neurotic symptom type according to level of perceived social support deficit $(\mathrm{N}=9708)$

\begin{tabular}{|c|c|c|c|c|}
\hline \multirow[b]{2}{*}{ Symptom type } & \multicolumn{3}{|c|}{ Perceived social support score grouped } & \multirow[b]{2}{*}{ Total } \\
\hline & Severe & Moderate & No lack & \\
\hline $\begin{array}{l}\text { Somatic symptc } \\
\text { Proportion } \\
95 \% \text { CI }\end{array}$ & $\begin{array}{c}0 \cdot 11 \\
0 \cdot 09-0 \cdot 14\end{array}$ & $\begin{array}{c}0 \cdot 08 \\
0 \cdot 07-0 \cdot 09\end{array}$ & $\begin{array}{c}0 \cdot 07 \\
0 \cdot 06-0 \cdot 07\end{array}$ & $\begin{array}{c}0.07 \\
0 \cdot 07-0.08\end{array}$ \\
\hline $\begin{array}{l}\text { Fatigue } \\
\text { Proportion } \\
95 \% \text { CI }\end{array}$ & $\begin{array}{c}0 \cdot 33 \\
0 \cdot 30-0 \cdot 37\end{array}$ & $\begin{array}{c}0 \cdot 28 \\
0 \cdot 26-0 \cdot 30\end{array}$ & $\begin{array}{c}0 \cdot 25 \\
0 \cdot 24-0 \cdot 27\end{array}$ & $\begin{array}{c}0 \cdot 27 \\
0 \cdot 26-0 \cdot 28\end{array}$ \\
\hline $\begin{array}{l}\text { Sleep problems } \\
\text { Proportion } \\
95 \% \text { CI }\end{array}$ & $\begin{array}{c}0 \cdot 31 \\
0 \cdot 28-0 \cdot 35\end{array}$ & $\begin{array}{c}0 \cdot 26 \\
0 \cdot 24-0 \cdot 28\end{array}$ & $\begin{array}{c}0 \cdot 23 \\
0 \cdot 22-0 \cdot 25\end{array}$ & $\begin{array}{c}0 \cdot 25 \\
0 \cdot 24-0 \cdot 26\end{array}$ \\
\hline $\begin{array}{l}\text { Irritability } \\
\text { Proportion } \\
95 \% \text { CI }\end{array}$ & $\begin{array}{c}0 \cdot 29 \\
0 \cdot 26-0 \cdot 32\end{array}$ & $\begin{array}{c}0 \cdot 26 \\
0 \cdot 24-0 \cdot 28\end{array}$ & $\begin{array}{c}0 \cdot 20 \\
0 \cdot 19-0 \cdot 21\end{array}$ & $\begin{array}{c}0 \cdot 22 \\
0 \cdot 21-0 \cdot 23\end{array}$ \\
\hline $\begin{array}{l}\text { Worry (excludi } \\
\text { Proportion } \\
95 \% \text { CI }\end{array}$ & $\begin{array}{c}\text { physical he: } \\
0 \cdot 30 \\
0 \cdot 26-0 \cdot 32\end{array}$ & $\begin{array}{c}0 \cdot 21 \\
0 \cdot 20-0 \cdot 23\end{array}$ & $\begin{array}{c}0 \cdot 18 \\
0 \cdot 17-0 \cdot 19\end{array}$ & $\begin{array}{c}0 \cdot 20 \\
0 \cdot 19-0 \cdot 21\end{array}$ \\
\hline $\begin{array}{l}\text { Depression } \\
\text { Proportion } \\
95 \% \text { CI }\end{array}$ & $\begin{array}{c}0 \cdot 21 \\
0 \cdot 18-0 \cdot 24\end{array}$ & $\begin{array}{c}0 \cdot 10 \\
0 \cdot 09-0 \cdot 12\end{array}$ & $\begin{array}{c}0 \cdot 08 \\
0 \cdot 07-0 \cdot 08\end{array}$ & $\begin{array}{c}0.09 \\
0.09-0 \cdot 10\end{array}$ \\
\hline $\begin{array}{l}\text { Depressive idea } \\
\text { Proportion } \\
95 \% \text { CI }\end{array}$ & $\begin{array}{c}0 \cdot 18 \\
0 \cdot 16-0 \cdot 21\end{array}$ & $\begin{array}{c}0 \cdot 11 \\
0 \cdot 09-0 \cdot 13\end{array}$ & $\begin{array}{c}0 \cdot 07 \\
0 \cdot 06-0 \cdot 08\end{array}$ & $\begin{array}{c}0.09 \\
0 \cdot 08-0 \cdot 10\end{array}$ \\
\hline $\begin{array}{l}\text { Anxiety } \\
\text { Proportion } \\
95 \% \text { CI }\end{array}$ & $\begin{array}{c}0 \cdot 17 \\
0 \cdot 14-0 \cdot 19\end{array}$ & $\begin{array}{c}0 \cdot 10 \\
0 \cdot 09-0 \cdot 11\end{array}$ & $\begin{array}{c}0 \cdot 08 \\
0.08-0 \cdot 09\end{array}$ & $\begin{array}{c}0.09 \\
0 \cdot 09-0 \cdot 10\end{array}$ \\
\hline $\begin{array}{l}\text { Obsessions } \\
\text { Proportion } \\
95 \% \text { CI }\end{array}$ & $\begin{array}{c}0 \cdot 14 \\
0 \cdot 12-0 \cdot 16\end{array}$ & $\begin{array}{c}0 \cdot 10 \\
0 \cdot 09-0 \cdot 11\end{array}$ & $\begin{array}{c}0 \cdot 08 \\
0.08-0.09\end{array}$ & $\begin{array}{c}0.09 \\
0.09-0 \cdot 10\end{array}$ \\
\hline $\begin{array}{l}\text { Concentration } \\
\text { Proportion } \\
95 \% \mathrm{CI}\end{array}$ & $\begin{array}{l}\text { ss } \\
\quad 0 \cdot 15 \\
0 \cdot 13-0 \cdot 18\end{array}$ & $\begin{array}{c}0 \cdot 09 \\
0 \cdot 08-0 \cdot 10\end{array}$ & $\begin{array}{c}0 \cdot 07 \\
0 \cdot 06-0 \cdot 07\end{array}$ & $\begin{array}{c}0.08 \\
0 \cdot 07-0 \cdot 09\end{array}$ \\
\hline $\begin{array}{l}\text { Compulsions } \\
\text { Proportion } \\
95 \% \text { CI }\end{array}$ & $\begin{array}{c}0 \cdot 08 \\
0 \cdot 07-0 \cdot 10\end{array}$ & $\begin{array}{c}0 \cdot 07 \\
0 \cdot 06-0 \cdot 08\end{array}$ & $\begin{array}{c}0 \cdot 06 \\
0.05-0 \cdot 06\end{array}$ & $\begin{array}{c}0.06 \\
0.06-0.07\end{array}$ \\
\hline $\begin{array}{l}\text { Phobias } \\
\text { Proportion } \\
95 \% \text { CI }\end{array}$ & $\begin{array}{c}0 \cdot 09 \\
0 \cdot 07-0 \cdot 11\end{array}$ & $\begin{array}{c}0 \cdot 06 \\
0 \cdot 05-0 \cdot 07\end{array}$ & $\begin{array}{c}0 \cdot 04 \\
0.04-0 \cdot 05\end{array}$ & $\begin{array}{c}0 \cdot 05 \\
0 \cdot 05-0 \cdot 06\end{array}$ \\
\hline $\begin{array}{l}\text { Worry about } \mathrm{p} \\
\text { Proportion } \\
95 \% \text { CI }\end{array}$ & $\begin{array}{c}0 \cdot 09 \\
0 \cdot 07-0 \cdot 11\end{array}$ & $\begin{array}{c}0.04 \\
0.03-0.05\end{array}$ & $\begin{array}{c}0 \cdot 04 \\
0 \cdot 04-0 \cdot 05\end{array}$ & $\begin{array}{c}0.05 \\
0.04-0.05\end{array}$ \\
\hline $\begin{array}{l}\text { Panic } \\
\text { Proportion } \\
95 \% \text { CI }\end{array}$ & $\begin{array}{c}0.06 \\
0.05-0 \cdot 08\end{array}$ & $\begin{array}{c}0 \cdot 02 \\
0 \cdot 02-0 \cdot 03\end{array}$ & $\begin{array}{c}0 \cdot 02 \\
0 \cdot 02-0 \cdot 03\end{array}$ & $\begin{array}{c}0.02 \\
0 \cdot 02-0 \cdot 03\end{array}$ \\
\hline $\begin{array}{c}\text { Total CIS-R > } \\
\text { Proportion } \\
95 \% \text { CI }\end{array}$ & $\begin{array}{c}0 \cdot 27 \\
0 \cdot 24-0 \cdot 30\end{array}$ & $\begin{array}{c}0 \cdot 15 \\
0 \cdot 14-0 \cdot 17\end{array}$ & $\begin{array}{c}0 \cdot 12 \\
0 \cdot 11-0 \cdot 14\end{array}$ & $\begin{array}{c}0 \cdot 15 \\
0 \cdot 14-0 \cdot 16\end{array}$ \\
\hline
\end{tabular}

associated with deficient social support (Tables 2 and 3) but the overall impression was of the similarity of associations with a deficiency in primary group size and perceived social support across symptom types (Tables 2 and 3).

Given the lack of firm and consistent associations with any specific type of neurotic symptom in univariate analyses, the total CIS-R score $(\geqslant 12$ versus fewer neurotic symptoms of any type) was used in logistic regression models (Table 4). There was a higher prevalence of neurotic disorder in those with a primary support group of $\leqslant 3$ (odds ratio, $2.4 ; 95 \%$ CI, $2 \cdot 0$ to $3 \cdot 0$ ) compared with a larger primary 
Table 4. Survey logistic regression on CIS-R total score $\geqslant 12$

\begin{tabular}{|c|c|c|c|c|}
\hline Predictor variable & OR & $95 \% \mathrm{CI}$ & Adjusted OR & $95 \% \mathrm{CI}$ \\
\hline Primary support group $\leqslant 3$ & $2 \cdot 43$ & $2 \cdot 01-2 \cdot 93$ & 1.89 & $1 \cdot 53-2 \cdot 33$ \\
\hline Perceived social support low ( $v$. adequate) & $2 \cdot 40$ & $2 \cdot 03-2 \cdot 84$ & $2 \cdot 06$ & $1 \cdot 7-2 \cdot 49$ \\
\hline Employment status: (unemployed $v . \mathrm{FT} / \mathrm{PT} /$ economically inactive) & 1.98 & $1 \cdot 64-2 \cdot 38$ & $1 \cdot 42$ & $1 \cdot 14-1 \cdot 76$ \\
\hline \multicolumn{5}{|l|}{ Family type: } \\
\hline Couple with no children & - & - & $1 \cdot 0$ & - \\
\hline Couple with children & $1 \cdot 21$ & $1 \cdot 03-1 \cdot 42$ & $1 \cdot 31$ & $1 \cdot 11-1 \cdot 55$ \\
\hline Lone parent with $\geqslant 1$ children & $2 \cdot 64$ & $2 \cdot 16-3 \cdot 23$ & $1 \cdot 80$ & $1 \cdot 42-2 \cdot 27$ \\
\hline One person in household & $1 \cdot 73$ & $1 \cdot 45-2 \cdot 05$ & $1 \cdot 47$ & $1 \cdot 20-1 \cdot 79$ \\
\hline Adult living with one parent & $0 \cdot 73$ & $0.54-0.99$ & $0 \cdot 81$ & $0 \cdot 59-1 \cdot 11$ \\
\hline Adult living with both parents & $1 \cdot 17$ & $0 \cdot 77-1 \cdot 79$ & $1 \cdot 10$ & $0 \cdot 69-1 \cdot 76$ \\
\hline Sex (female $v$. male) & 1.79 & $1 \cdot 58-2 \cdot 03$ & $1 \cdot 91$ & $1 \cdot 66-2 \cdot 21$ \\
\hline No use of car & 1.95 & $1 \cdot 70-2 \cdot 23$ & $1 \cdot 31$ & $1 \cdot 11-1 \cdot 56$ \\
\hline No longstanding illness of disability & $0 \cdot 32$ & $0 \cdot 28-0 \cdot 37$ & $0 \cdot 40$ & $0 \cdot 30-0 \cdot 39$ \\
\hline Any threatening life event in past year ( $v$. none) & $2 \cdot 61$ & $2 \cdot 27-2 \cdot 99$ & $2 \cdot 35$ & $2 \cdot 04-2 \cdot 72$ \\
\hline
\end{tabular}

Number of observations $9837 ; F 12,173=64 \cdot 6 ; P=0 \cdot 0000$.

group; the odds ratio for severe lack of perceived social support was $2 \cdot 4(95 \% \mathrm{CI}, 2 \cdot 0$ to $2 \cdot 8)$. The association with social support deficit was hardly affected by including in an adjusted logistic model other factors that might explain the association with deficient social support, including unemployment, an index of poverty (lack of car ownership), reporting a threatening life event in the previous 6 months, living as a single parent with one or more children, female gender and information on long-term physical health problems (Table 4). After controlling for these potential confounders, respondents with a severe deficit in perceived social support were still more than twice as likely to have a high CIS-R score (Table 4). Respondents with a primary support group of $\leqslant 3$ were just under twice as likely to have a high CIS-R score when compared with those with a larger primary group (Table 4). Additional analysis also showed that the association was not altered by taking into account living in an urban area and difficulties with carrying out activities of daily living, which are strongly associated with neurotic disorder (Bebbington et al. 2000; Paykel et al. 2000).

Table 5 presents the estimated odds ratios and $95 \%$ confidence intervals for six multivariate Huber logistic models. Models 1 and 2 are unadjusted for any other covariates. Models 3 and 4 are adjusted for low perceived social support/small primary support group, unemployment, sex, family unit type, no use of car, no longstanding illness and any threatening life events in past year. Models 5 and 6 are adjusted for low perceived social support/small primary support group, living in an urban area, difficulties in carrying out activities of daily living, unemployment, sex, family unit type, no use of car, no longstanding illness and any threatening life events in past year.

As the effects of primary support group differed by symptom (i.e. interactions between primary support group size and symptoms were significant at the $5 \%$ level), separate effects were estimated for each symptom; these results are presented in models 1,3 and 5 in Table 5.

Associations between primary support group size and neurotic symptoms varied by symptom type. In Model 1, a significant interaction between primary support group size and symptom type indicated higher prevalence of somatic symptoms, problems with concentration/forgetfulness, worrying about physical health, depression, depressive ideas, anxiety, phobias and panic symptoms, for subjects with a primary group size of $\leqslant 3$ compared with subjects with a larger primary support group. Other than phobias, these findings were not affected by adjustment for low perceived social support, unemployment, sex, family unit type, no use of car, no longstanding illness and any threatening life events in past year (Table 5, Model 3). When adjustments were made for low perceived social support, living in an urban area, difficulties in carrying out activities of daily living, unemployment, sex, family unit type, no use of car, no longstanding illness and any threatening life events in past year (Model 5), higher prevalence of problems with concentration/forgetfulness, depression, depressive ideas, anxiety and panic 
Table 5. Odds Ratio (95\% CI) for multivariate Huber logistic models of each symptom type taking account of each other symptom type

\begin{tabular}{|c|c|c|c|c|c|c|c|c|c|c|c|c|c|c|}
\hline & Somatic & Fatigue & $\begin{array}{l}\text { Conc/ } \\
\text { Forget }\end{array}$ & Sleep & Irritability & $\begin{array}{l}\text { Worry/ } \\
\text { Physical } \\
\text { health }\end{array}$ & Depression & $\begin{array}{l}\text { Depressive } \\
\text { ideas }\end{array}$ & Worry & Anxiety & Phobias & Panic & Compulsion & Obsessions \\
\hline \multicolumn{15}{|l|}{ Models 1 and 2} \\
\hline $\begin{array}{l}\text { Primary support } \\
\text { group } \leqslant 3\end{array}$ & $\begin{array}{c}1 \cdot 61 \\
(1 \cdot 28,2 \cdot 00)^{*}\end{array}$ & $\begin{array}{c}1 \cdot 34 \\
(0 \cdot 86,2 \cdot 08)\end{array}$ & $\begin{array}{c}2 \cdot 22 \\
(1 \cdot 38,3 \cdot 56)^{*}\end{array}$ & $\begin{array}{c}1.39 \\
(0.87,2 \cdot 22)\end{array}$ & $\begin{array}{c}1 \cdot 38 \\
(0 \cdot 87,2 \cdot 17)\end{array}$ & $\begin{array}{c}2 \cdot 16 \\
(1 \cdot 32,3 \cdot 54)^{*}\end{array}$ & $\begin{array}{c}2 \cdot 69 \\
(1 \cdot 68,4 \cdot 29)^{*}\end{array}$ & $\begin{array}{c}2 \cdot 42 \\
(1 \cdot 52,3 \cdot 87)^{*}\end{array}$ & $\begin{array}{c}1 \cdot 69 \\
(1 \cdot 08,2 \cdot 65)\end{array}$ & $\begin{array}{c}2 \cdot 10 \\
(1 \cdot 32,3 \cdot 32)^{*}\end{array}$ & $\begin{array}{c}1 \cdot 99 \\
(1 \cdot 17,3 \cdot 37)^{*}\end{array}$ & $\begin{array}{c}3 \cdot 13 \\
(1 \cdot 80,5 \cdot 42)^{*}\end{array}$ & $\begin{array}{c}1 \cdot 41 \\
(0 \cdot 83,2 \cdot 39)\end{array}$ & $\begin{array}{c}1 \cdot 54 \\
(0 \cdot 94,2 \cdot 52)\end{array}$ \\
\hline $\begin{array}{l}\text { Perceived social } \\
\text { support low } \\
(v . \text { adequate })\end{array}$ & $\begin{array}{c}1 \cdot 65 \\
(1 \cdot 28,2 \cdot 12)^{*}\end{array}$ & $\begin{array}{c}1.56 \\
(0 \cdot 94,2 \cdot 59)\end{array}$ & $\begin{array}{c}2 \cdot 45 \\
(1 \cdot 42,4 \cdot 23)^{*}\end{array}$ & $\begin{array}{c}1 \cdot 32 \\
(0 \cdot 78,2 \cdot 21)\end{array}$ & $\begin{array}{c}1 \cdot 45 \\
(0 \cdot 86,2 \cdot 42)\end{array}$ & $\begin{array}{c}1 \cdot 89 \\
(1 \cdot 05,3 \cdot 41)\end{array}$ & $\begin{array}{c}2 \cdot 82 \\
(1 \cdot 63,4 \cdot 84)^{*}\end{array}$ & $\begin{array}{c}2 \cdot 68 \\
(1 \cdot 55,4 \cdot 62)^{*}\end{array}$ & $\begin{array}{c}1.66 \\
(0.99,2 \cdot 76)\end{array}$ & $\begin{array}{c}2 \cdot 27 \\
(1 \cdot 33,3 \cdot 85)^{*}\end{array}$ & $\begin{array}{c}2 \cdot 35 \\
(1 \cdot 30,4 \cdot 25)^{*}\end{array}$ & $\begin{array}{c}3 \cdot 27 \\
(1 \cdot 72,6 \cdot 20)^{*}\end{array}$ & $\begin{array}{c}2 \cdot 05 \\
(1 \cdot 14,3 \cdot 66)^{*}\end{array}$ & $\begin{array}{c}1 \cdot 67 \\
(0 \cdot 94,2 \cdot 93)\end{array}$ \\
\hline \multicolumn{15}{|l|}{ Models 3 and 4} \\
\hline $\begin{array}{l}\text { Primary support } \\
\text { group } \leqslant 3\end{array}$ & $\begin{array}{c}1 \cdot 41 \\
(1 \cdot 12,1 \cdot 75)^{*}\end{array}$ & $\begin{array}{c}1 \cdot 21 \\
(0 \cdot 76,1 \cdot 89)\end{array}$ & $\begin{array}{c}1 \cdot 98 \\
(1 \cdot 22,3 \cdot 20)^{*}\end{array}$ & $\begin{array}{c}1 \cdot 25 \\
(0 \cdot 77,2 \cdot 02)\end{array}$ & $\begin{array}{c}1 \cdot 23 \\
(0 \cdot 77,1 \cdot 97)\end{array}$ & $\begin{array}{c}1 \cdot 89 \\
(1 \cdot 14,3 \cdot 13)^{*}\end{array}$ & $\begin{array}{c}2 \cdot 43 \\
(1 \cdot 50,3 \cdot 92)^{*}\end{array}$ & $\begin{array}{c}2 \cdot 17 \\
(1 \cdot 34,3 \cdot 50)^{*}\end{array}$ & $\begin{array}{c}1 \cdot 53 \\
(0 \cdot 96,2 \cdot 42)\end{array}$ & $\begin{array}{c}1 \cdot 87 \\
(1 \cdot 16,2 \cdot 99)^{*}\end{array}$ & $\begin{array}{c}1 \cdot 74 \\
(1 \cdot 01,2 \cdot 98)\end{array}$ & $\begin{array}{c}2 \cdot 72 \\
(1 \cdot 55,4 \cdot 76)^{*}\end{array}$ & $\begin{array}{c}1 \cdot 23 \\
(0 \cdot 71,2 \cdot 10)\end{array}$ & $\begin{array}{c}1 \cdot 35 \\
(0 \cdot 81,2 \cdot 23)\end{array}$ \\
\hline $\begin{array}{l}\text { Perceived social } \\
\text { support low } \\
(v . \text { adequate })\end{array}$ & $\begin{array}{c}1 \cdot 30 \\
(1 \cdot 00,1 \cdot 68)\end{array}$ & $\begin{array}{c}1 \cdot 28 \\
(0 \cdot 76,2 \cdot 16)\end{array}$ & $\begin{array}{c}1.97 \\
(1 \cdot 12,3 \cdot 46)^{*}\end{array}$ & $\begin{array}{c}1 \cdot 06 \\
(0 \cdot 62,1 \cdot 81)\end{array}$ & $\begin{array}{c}1 \cdot 17 \\
(0 \cdot 68,2 \cdot 00)\end{array}$ & $\begin{array}{c}1 \cdot 49 \\
(0 \cdot 81,2 \cdot 73)\end{array}$ & $\begin{array}{c}2 \cdot 30 \\
(1 \cdot 31,4 \cdot 02)^{*}\end{array}$ & $\begin{array}{c}2 \cdot 17 \\
(1 \cdot 23,3 \cdot 81)^{*}\end{array}$ & $\begin{array}{c}1 \cdot 35 \\
(0 \cdot 79,2 \cdot 28)\end{array}$ & $\begin{array}{c}1 \cdot 83 \\
(1 \cdot 05,3 \cdot 16)\end{array}$ & $\begin{array}{c}1 \cdot 87 \\
(1 \cdot 01,3 \cdot 44)\end{array}$ & $\begin{array}{c}2 \cdot 58 \\
(1 \cdot 33,4 \cdot 97)^{*}\end{array}$ & $\begin{array}{c}1 \cdot 63 \\
(0 \cdot 89,2 \cdot 97)\end{array}$ & $\begin{array}{c}1 \cdot 32 \\
(0 \cdot 73,2 \cdot 32)\end{array}$ \\
\hline \multicolumn{15}{|l|}{ Models 5 and 6} \\
\hline $\begin{array}{l}\text { Primary support } \\
\text { group } \leqslant 3\end{array}$ & $\begin{array}{c}1 \cdot 32 \\
(1 \cdot 05,1 \cdot 65)\end{array}$ & $\begin{array}{c}1 \cdot 15 \\
(0 \cdot 73,1 \cdot 81)\end{array}$ & $\begin{array}{c}1 \cdot 88 \\
(1 \cdot 15,3 \cdot 05)^{*}\end{array}$ & $\begin{array}{c}1 \cdot 19 \\
(0 \cdot 73,1 \cdot 93)\end{array}$ & $\begin{array}{c}1 \cdot 17 \\
(0 \cdot 73,1 \cdot 88)\end{array}$ & $\begin{array}{c}1 \cdot 78 \\
(1 \cdot 07,2 \cdot 95)\end{array}$ & $\begin{array}{c}2 \cdot 32 \\
(1 \cdot 43,3 \cdot 76)^{*}\end{array}$ & $\begin{array}{c}2 \cdot 07 \\
(1 \cdot 27,3 \cdot 34)^{*}\end{array}$ & $\begin{array}{c}1 \cdot 46 \\
(0 \cdot 92,2 \cdot 32)\end{array}$ & $\begin{array}{c}1 \cdot 78 \\
(1 \cdot 10,2 \cdot 86)^{*}\end{array}$ & $\begin{array}{c}1 \cdot 63 \\
(0 \cdot 94,2 \cdot 81)\end{array}$ & $\begin{array}{c}2.55 \\
(1 \cdot 45,4 \cdot 47)^{*}\end{array}$ & $\begin{array}{c}1.15 \\
(0.66,1.97)\end{array}$ & $\begin{array}{c}1 \cdot 27 \\
(0 \cdot 76,2 \cdot 12)\end{array}$ \\
\hline $\begin{array}{l}\text { Perceived social } \\
\text { support low } \\
(v . \text { adequate })\end{array}$ & $\begin{array}{c}1 \cdot 23 \\
(0 \cdot 95,1 \cdot 60)\end{array}$ & $\begin{array}{c}1 \cdot 24 \\
(0 \cdot 72,2 \cdot 11)\end{array}$ & $\begin{array}{c}1 \cdot 89 \\
(1 \cdot 06,3 \cdot 36)\end{array}$ & $\begin{array}{c}1 \cdot 02 \\
(0 \cdot 58,1 \cdot 76)\end{array}$ & $\begin{array}{c}1 \cdot 12 \\
(0 \cdot 65,1 \cdot 94)\end{array}$ & $\begin{array}{c}1 \cdot 41 \\
(0 \cdot 76,2 \cdot 62)\end{array}$ & $\begin{array}{c}2 \cdot 21 \\
(1 \cdot 24,3 \cdot 92)^{*}\end{array}$ & $\begin{array}{c}2 \cdot 09 \\
(1 \cdot 17,3 \cdot 71)^{*}\end{array}$ & $\begin{array}{c}1 \cdot 29 \\
(0 \cdot 75,2 \cdot 22)\end{array}$ & $\begin{array}{c}1 \cdot 75 \\
(0 \cdot 99,3 \cdot 06)\end{array}$ & $\begin{array}{c}1 \cdot 78 \\
(0 \cdot 95,3 \cdot 32)\end{array}$ & $\begin{array}{c}2 \cdot 45 \\
(1 \cdot 25,4 \cdot 77)^{*}\end{array}$ & $\begin{array}{c}1 \cdot 55 \\
(0 \cdot 84,2 \cdot 87)\end{array}$ & $\begin{array}{c}1 \cdot 26 \\
(0 \cdot 69,2 \cdot 29)\end{array}$ \\
\hline
\end{tabular}

Models 1 and 2: unadjusted for any other covariates.

Models 3 and 5: adjusted for low perceived social support/small primary support group, unemployment, sex, family unit type, no use of car, no longstanding illness and any threatening life events in past year.

Models 5 and 6: adjusted for low perceived social support/small primary support group, living in an urban area, difficulties in carrying out activities of daily living, unemployment, sex, family unit type, no use of car, no longstanding illness and any threatening life events in past year.

$$
* P<0.05 \text {. }
$$


symptoms were indicated for subjects with a primary group size of $\leqslant 3$ compared with subjects with a larger primary support group.

As the effects of perceived social support differed by symptom (i.e. interactions between perceived social support and symptoms were significant at the $5 \%$ level), separate effects were estimated for each symptom; these results are presented in models 2, 4 and 6 in Table 5.

Associations between perceived social support and neurotic symptoms also varied by symptom type. In Model 2, a significant interaction between perceived social support and symptom type indicated higher prevalence of somatic symptoms, problems with concentration/forgetfulness, depression, depressive ideas, anxiety, phobias, panic symptoms and compulsions, for subjects with low perceived social support compared with subjects with adequate perceived social support. Other than somatic symptoms, anxiety, phobias and compulsions, these findings were not affected by adjustment for small primary support group, unemployment, sex, family unit type, no use of car, no longstanding illness and any threatening life events in past year (Model 4). When adjustments were made for small primary support group, living in an urban area, difficulties in carrying out activities of daily living, unemployment, sex, family unit type, no use of car, no longstanding illness and any threatening life events in past year (Model 6), higher prevalence of depression, depressive ideas and panic symptoms were indicated for subjects with low perceived social support compared with subjects with adequate perceived social support.

In summary, depression, depressive ideas and panic symptoms have higher prevalence (significant) in subjects with deficits in social support networks, in all six models. No other interactions between symptoms and other sociodemographic predictors were examined.

Logistic regression models were also used to determine predictors of social support deficits. We report here only associations with probability values $<1 \%$ (detailed tables available on request). Having a primary group size of $\leqslant 3$ was found to be predicted by male gender, longterm physical ill health, lack of car ownership and living either as a single parent or as an adult living with both parents only. Neither unemployment nor recent stressful life events were associated. However, a severe deficit in perceived social support was associated with all of these variables. It was also associated with all family unit types apart from living as a couple (with or without children).

\section{DISCUSSION}

In this study, using an appropriate multivariate statistical model, we have shown that the association between deficits in social support and neurotic symptoms is better explained by associations conditional upon the type of symptom assessed. We have also been able to identify factors associated with deficits in social support that might be informative in designing risk factor reduction programmes to prevent specific types of neurotic symptoms in the community, depression and depressive ideas being the most consistently predicted by low social support.

A number of study limitations need to be considered. First, no causal inference can be made of the association of social support deficits with specific types of neurotic symptoms reported here. Clearly longitudinal research within such samples is required in order to better understand these findings, including examining the alternative conclusion that such symptoms cause low support. Elsewhere we report findings from an earlier national survey (Grainge et al. 2000) in men assessed prospectively in which the same measure of perceived social support predicted respondents' overall depressive symptom levels 7 years later even when prior symptoms were controlled for in the analysis; among women the effect of perceived social support was weaker and possibly attributable to the play of chance. We discuss other relevant longitudinal studies of different indices of social support below. However, we are encouraged in thinking that at least some important element of the direction of causality is from social risk factors to symptoms because our findings stand up when we control for other environmental and individual factors that would be expected to act as confounders, such as difficulties in carrying out activities of daily living and the presence of longstanding illness (Table 5) both of which would be expected to reduce the ability of an individual to maintain their support system. 
A second possible limitation in the present study was that only self-report measures of risk factors and neurotic symptoms were used, albeit administered by experienced survey interviewers. The utility of such self-report methods has been investigated very little. When genetic and environmental risk factors for depression in the preceding month were examined in a comparison of the semi-structured SCID-I and self report SCL in female twins the between twin monozygotic and dizygotic correlations for depression were 0.26 and 0.03 for the SCL and 0.43 and 0.19 for the SCID (Foley et al. 2001). When Andrews \& Brown (1993) compared two measures of low self-esteem in a study of depression in women, the investigatorrated Self-Esteem and Social Support scales (SESS) and a self-report measure, they found that the SESS accounted for unique variance when the two measures were considered together and current depression was controlled (Andrews \& Brown, 1993). With regard to our findings firmer conclusions might be possible by integrating information from the present self-report measures with data provided by instruments employing clinical ratings or investigator judgements (Brugha et al. $1999 b)$.

However, we have been able to address a further major potential methodological limitation by appropriately modelling predictors of non-independent multiple dependent (or outcome) variables. This method has only been applied previously on one occasion to our knowledge and in a somewhat simpler case to identify factors associated with service use for child psychopathology in just three settings: mental health, general health, and school (Zahner \& Daskalakis, 1997; Horton et al. 1999). Had we not made use of this method we could have reached the misleading conclusion that our social risk factors predict all neurotic symptom types essentially equivalently as Tables 2 and 3 appear to suggest. The findings in Table 5 point interestingly to the conclusion that deficits in social network size and social support predict specifically psychological/cognitive aspects of neurotic disorder and not its somatic and physical health correlates. This would seem to fit with theory suggesting that the cognitive and not the somatic aspects of depression are predicted by social risk factors. However, we have yet to examine this in relation to other risk measures of interest to us such as stressful life events (Brugha \& Conroy, 1985; Bebbington et al. 1988).

A previous report from the present survey found that depressive symptoms were not specifically associated with socio-economic indicators after adjustment for the total score on the CIS-R (Lewis et al. 1998). This is in contrast, for example, with work suggesting that life events involving loss and danger respectively are more likely to be associated with depressive and anxiety disorders respectively (Finlay-Jones \& Brown, 1981; Brown et al. 1992, 1995). However, neither of these earlier studies made use of the modelling specifications employed here (Table 5).

Comment is also warranted by our finding that, of our two indices of social support, the size of the close social network ("primary group support' (Table 5)) is significantly associated with a slightly wider range of symptom types than the presumably more subjective index of perceived social support. Surprisingly, the simple to collect variable primary group size has been of little interest to other social support researchers over the past three decades (Brugha, 1988; Brugha et al. 1998). The importance of perceived or subjective social support has been endorsed in a wide range of social support studies, but few researchers have focused on the size of the person's primary social network. Nevertheless, as in this study, our work repeatedly shows that these two variables function independently of one another. Two early casecontrol studies (Henderson et al. 1978; Brugha et al. 1982) were followed by other studies showing the importance of network size in predicting recovery from depression (Brugha et al. $1990 \mathrm{~b}$ ) but curiously not in the onset of postnatal depression (Brugha et al. 1998). We hope that other researchers will be encouraged to investigate this further. A clue to possible future directions for this work came from a study that showed that primary group size predicts recovery not in a first or second episode but in later episodes of depression (Brugha et al. 1997). One possible explanation put forward was that adults who had recurrent depressive episodes will perforce have learnt who they can truly rely upon as a source of close support; it may be that their replies to such a social 
network interview generate more valid information about the construct.

Environmental and individual influences both need to be considered in researching the 'causes' of social support including developmental (Champion, 1995), and cognitive aspects (Brewin, 1995). If longitudinal research supports the suggestion that levels of social support predict specific types of neurotic symptoms, this should be followed by the development of interventions that could then be evaluated using experimental designs (Brugha et al. 2000). The development of effective risk reduction interventions is likely to depend on the identification of malleable risk and protective factors (Mrazek \& Haggerty, 1994) and of their causes. Two related factors appear to be associated with the social support deficits found in the present study: poverty; and, living in a household either alone or in the role of a single carer. Both of these factors might be amenable to action at different levels of input ranging from individual to community support and the provision of material and welfare support at a wider societal level. But from the point of view of the prevention of future neurotic symptoms, the present data do suggest that the development of interventions designed to improve social support could turn out to operate differently in relation to specific types of symptoms. Thus, depression and possibly generalized anxiety and panic appear to be associated with social support deficits but not phobias or obsessive-compulsive phenomena suggesting that different risk factors and mechanisms apply to those forms of psychopathology (Table 5). Both theoretical and empirical research will be needed therefore.

In conclusion, highly significant associations have been found between specific types of neurotic symptoms, particularly depressive symptoms, and social network variables, and these are not removed when account is taken of other psychosocial confounders. Information about the possible causes of social support deficits should inform the development of risk reducing interventions at the population and individual level. The development and evaluation of such interventions may lead to better informed prevention policies and improved mental health in the general population.

\section{REFERENCES}

Andrews, B. \& Brown, G. W. (1993). Self-esteem and vulnerability to depression: the concurrent validity of interview and questionnaire measures. Journal of Abnormal Psychology 102, 565-572.

Bebbington, P., Brugha, T., Meltzer, H., Farrell, M., Ceresa, C., Jenkins, R. \& Lewis, G. (2000). Psychiatric disorder and dysfunction in the UK national survey of psychiatric morbidity. Social Psychiatry and Psychiatric Epidemiology 35, 191-197.

Bebbington, P. E., Brugha, T., MacCarthy, B., Potter, J., Sturt, E., Wykes, T., Katz, R. \& McGuffin, P. (1988). The Camberwell Collaborative Depression Study. I. Depressed probands: adversity and the form of depression. British Journal of Psychiatry 152, 754-765.

Breeze, E., Maidment, A., Bennett, N., Flatley, J. \& Carey, S. (1994). Health Survey for England 1992. HMSO: London.

Brewin, C. R. (1995). Cognitive aspects of social support processes. In Social Support and Psychiatric Disorder: Research Findings and Guidelines for Clinical Practice (ed. T. S. Brugha), pp. 96-116. Cambridge University Press: Cambridge.

Brown, G. W., Andrews, B., Harris, T., Adler, Z. \& Bridge, L. (1986). Social Support, self-esteem and depression. Psychological Medicine 16, 813-831.

Brown, G. W., Lemyre, L. \& Bifulco, A. (1992). Social factors and recovery from anxiety and depressive disorders: a test of specificity. British Journal of Psychiatry 161, 1-54.

Brown, G. W., Harris, T. O. \& Hepworth, C. (1995). Loss, humiliation and entrapment among women developing depression: a patient and non patient sample. Psychological Medicine $\mathbf{2 5}$ 7-21

Brugha, T. (1988). Social Psychiatry. In The Instruments of Psychiatric Research (ed. C. Thompson), pp. 253-270. Wiley: Chichester.

Brugha, T. S. (1995). Social support and psychiatric disorder: overview of evidence. In Social Support and Psychiatric Disorder. Research Findings and Guidelines for Clinical Practice (ed. T. S. Brugha), pp. 1-40. Cambridge University Press: Cambridge.

Brugha, T.S. \& Conroy, R. (1985). Categories of depression: reported life events in a controlled design. British Journal of Psychiatry 147, 641-646.

Brugha, T., Conroy, R., Walsh, N., Delaney, W., O'Hanlon, J., Dondero, E., Daly, L., Hickey, N. \& Bourke, G. (1982). Social networks, attachments and support in minor affective disorders: a replication. British Journal of Psychiatry 141, 249-255.

Brugha, T., Bebbington, P., Tennant, C. \& Hurry, J. (1985). The List of Threatening Experiences: a subset of 12 life event categories with considerable long-term contextual threat. Psychological Medicine 15, 189-194.

Brugha, T. S., Sturt, E., MacCarthy, B., Potter, J., Wykes, T. \& Bebbington, P. E. (1987). The Interview Measure of Social Relationships: the description and evaluation of a survey instrument for assessing personal social resources. Social Psychiatry 22, 123-128.

Brugha, T.S., Bebbington, P. E., Sturt, E., MacCarthy, B. \& Wykes, T. $(1990 a)$. The relation between life events and social support networks in a clinically depressed cohort. Social Psychiatry and Psychiatric Epidemiology 25, 308-313.

Brugha, T. S., Bebbington, P. E., MacCarthy, B., Sturt, E., Wykes, T. \& Potter, J. (1990 b). Gender, social support and recovery from depressive disorders: a prospective clinical study. Psychological Medicine 20, 147-156.

Brugha, T. S., Wing, J. K., Brewin, C. R., MacCarthy, B. \& Lesage, A. (1993). The relationship of social network deficits with deficits in social functioning in long-term psychiatric disorders. Social Psychiatry and Psychiatric Epidemiology 28, 218-224.

Brugha, T. S., Bebbington, P. E., Stretch, D. D., MacCarthy, B. \& Wykes, T. (1997). Predicting the short-term outcome of first episodes and recurrences of clinical depression: a prospective study of life events, difficulties, and social support networks. Journal of Clinical Psychiatry 58, 298-306. 
Brugha, T. S., Sharp, H. M., Cooper, S. A., Weisender, C., Britto, D. Shinkwin, R., Sherrif, T. \& Kirwan, P. H. (1998). The Leicester 500 Project. Social support and the development of postnatal depressive symptoms, a prospective cohort survey. Psychological Medicine 28, 63-79.

Brugha, T. S., Bebbington, P., Jenkins, R., Meltzer, H., Taub, N. A., Janas, M. \& Vernon, J. (1999 a). Cross validation of a household population survey diagnostic interview: a comparison of CIS-R with SCAN ICD-10 diagnostic categories. Psychological Medicine 29, 1029-1042.

Brugha, T. S., Bebbington, P. E. \& Jenkins, R. (1999 b). A difference that matters: comparisons of structured and semi-structured diagnostic interviews of adults in the general population. Psychological Medicine 29, 1013-1020.

Brugha, T. S., Wheatley, S., Taub, N. A., Culverwell, A., Freidman, T., Kirwan, P. H., Jones, D. R. \& Shapiro, D. A. (2000). Pragmatic randomized trial of antenatal intervention to prevent postnatal depression by reducing psychosocial risk factors. Psychological Medicine 30, 1273-1281.

Cassel, J. (1976). The contribution of the social environment to host resistance. American Jouranl of Epidemiology 104, 107-123.

Champion, L. (1995). A developmental perspective on social support networks. In Social Support and Psychiatric Disorder: Research Findings and Guidelines for Clinical Practice (ed. T. S. Brugha), pp. 61-95. Cambridge University Press: Cambridge.

Cobb, S. (1976). Social support as a moderator of life stress. Psychosomatic Medicine 38, 300-314.

Cox, B. D., Blaxter, M., Buckle, A. L. J., Fenner, N. P., Golding, J. F., Gore, M., Huppert, F. A., Nickson, J., Roth, M., Stark, J., Wadsworth, M. E. J. \& Wichelow, M. (1987). The Health and Lifestyle Survey. Health Promotion Research Trust: London.

Finlay-Jones, R. \& Brown, G. W. (1981). Types of stressful life event and the onset on anxiety and depressive disorders. Psychological Medicine 11, 803-816.

Foley, D. L., Neale, M. C. \& Kendler, K. S. (2001). Genetic and environmental risk factors for depression assessed by subject-rated Symptom Check List versus Structured Clinical Interview. Psychological Medicine 31, 1413-1423.

Goldberg, D. \& Huxley, P. (1992). A dimensional model for neurosis. In Common Mental Disorders. A Bio-social Model (ed. D. Goldberg and P. Huxley), pp. 65-81. Routledge: London.

Goldberg, D. P., Cooper, B., Eastwood, M. R., Kedward, H. B. \& Sheperd, M. (1970). A standardised psychiatric interview for use in community surveys. British Journal of Preventive and Social Medicine 24, 18-23.

Grainge, M. J., Brugha, T. S. \& Spiers, N. A. (2000). Social support, personality and depressive symptoms over 7 years: the Health and Lifestyle cohort. Social Psychiatry and Psychiatric Epidemiology 35, 366-374.

Harris, T., Brown, G. W. \& Robinson, R. (1999). Befriending as an intervention for chronic depression among women in an inner city. 1: Randomised controlled trial. British Journal of Psychiatry 174, 219-224.

Henderson, S. (1977). The social network, support and neurosis. British Journal of Psychiatry 131, 185-191.

Henderson, S., Duncan Jones, P., McAuley, H. \& Ritchie, K. (1978). The patient's primary group. British Journal of Psychiatry 132, $1-86$.

Horton, N. J., Bebchuk, J. D., Jones, C. L., Lipsitz, S. R., Catalano, P. J., Zahner, G. E. \& Fitzmaurice, G. M. (1999). Goodness-of-fit for GEE: an example with mental health service utilization. Statistical Medicine 18, 213-222.

House, J. S., Landis, K. R. \& Umberson, D. (1988). Social relationship and health. Science 241, 540-545.

Jenkins, R., Lewis, G., Bebbington, P., Brugha, T., Farrell, M., Gill, B. \& Meltzer, H. (1997 a). The national psychiatric morbidity surveys of Great Britain - initial findings from the household survey. Psychological Medicine 27, 775-789.

Jenkins, R., Bebbington, P., Brugha, T., Farrell, M., Gill, B., Lewis, G., Meltzer, H. \& Petticrew, M. (1997 b). The national psychiatric morbidity surveys of Great Britain-strategy and methods. Psychological Medicine 27, 765-774.

Kish, L. (1965). Survey Sampling. John Wiley \& Sons Ltd: London. Lewis, G., Pelosi, A. J., Araya, R. \& Dunn, G. (1992). Measuring psychiatric disorder in the community: a standardized assessment for use by lay interviewers. Psychological Medicine 22, 465-486.

Lewis, G., Bebbington, P., Brugha, T., Farrell, M., Gill, B., Jenkins, R. \& Meltzer, H. (1998). Socioeconomic status, standard of living and neurotic disorder. Lancet 352, 605-608.

Meltzer, H. \& Gill, B. (ed.) (1993). Office of Population Censuses \& Surveys Social Survey Division. OPCS Surveys of Psychiatric Morbidity in Great Britain. Report of a pilot study conducted among adults living in private households. Office of Population Censuses \& Surveys Social Survey Division: London.

Meltzer, H., Gill, B., Petticrew, M. \& Hinds, K. (ed.) (1995a). Office of Population Censuses \& Surveys Social Survey Division. OPCS Surveys of Psychiatric Morbidity in Great Britain. Report 1: The prevalence of psychiatric morbidity among adults living in private households. Appendix B. Algorithms for production of ICD-10 diagnoses of neurosis from the CIS-R. OPCS Surveys of Psychiatric Morbidity in Great Britain. Office for National Statistics Social Survey Division, HMSO: London.

Meltzer, H., Gill, B., Petticrew, M. \& Hinds, K. (ed.) (1995 b). Office of Population Censuses \& Surveys Social Survey Division. OPCS Surveys of Psychiatric Morbidity in Great Britain. Report 2: Physical complaints, service use and treatment of adults with psychiatric disorder. OPCS Surveys of Psychiatric Morbidity in Great Britain, HMSO: London.

Meltzer, H., Gill, B., Petticrew, M. \& Hinds, K. (ed.) (1995 c). Office of Population Censuses \& Surveys Social Survey Division. OPCS Surveys of Psychiatric Morbidity in Great Britain. Report 3: Economic activity and social functioning of adults with psychiatric disorders. OPCS Surveys of Psychiatric Morbidity in Great Britain, HMSO: London.

Mrazek, P. J. \& Haggerty, R. J. (1994). Reducing Risks for Mental Disorders: Frontiers for Preventive Intervention Research. National Academy of Medicine: Washington DC.

Paykel, E. S., Abbott, R. D., Jenkins, R., Brugha, T. \& Meltzer, H. (2000). Urban-rural mental health differences in Great Britain: findings from the National Morbidity Survey. Psychological Medicine 30, 269-280.

STATA Corp. (1999). Stata Statistical Software, Release 6.0. STATA Corp.: College Station, TX.

Tolsdorf, C.C. (1976). Social networks, support and coping: an exploratory study. Family Process 15, 407-417.

Wilson, P. R. \& Elliot, D. J. (1987). An evaluation of the Postcode Address File as a sampling frame and its use within OPCS. Journal of the Royal Statistical Society Series A (General) 150, 230-240.

Zahner, G. E. \& Daskalakis, C. (1997). Factors associated with mental health, general health, and school-based service use for child psychopathology. American Journal of Health 87, 1440-1448. 